- Maximize capacity to fluctuating vaccine allotments

Methods Our work leveraged several QI tools such as value stream mapping (figure 1), weighted average cycle times (figures 2 and 3), visual management (figure 4), and standard work. We utilized the Model for Improvement and sequential PDSA cycles (figure 5):

- Space required ongoing adjustments in roles, staffing, and flow. We cross-trained to allow for multi-skilled operation, and to accommodate social distancing through the entire process.

- To reflect our community's needs, we continuously adjusted to provide a mixed model of first/second dose, delivering

\begin{tabular}{|c|c|c|c|c|c|c|}
\hline & \begin{tabular}{|c|}
600 \\
patients. \\
12 hours. \\
\end{tabular} & \begin{tabular}{|c|}
600 \\
patients. \\
10 hours. \\
\end{tabular} & \begin{tabular}{|c|}
800 \\
patients. \\
12 hours. \\
\end{tabular} & \begin{tabular}{|c|}
800 \\
patients. \\
10 hours. \\
\end{tabular} & $\begin{array}{c}1000 \\
\text { patients. } \\
12 \text { hours } \\
\end{array}$ & $\begin{array}{c}1000 \\
\text { patients. } \\
10 \text { hours. }\end{array}$ \\
\hline Lead Time (min) & 25.5 & 25.5 & 25.5 & 25.5 & 25.5 & 25.5 \\
\hline Demand & 600 & 600 & 800 & 800 & 1000 & 1000 \\
\hline Length of Operation (min & 720 & 600 & 720 & 600 & 720 & 600 \\
\hline Takt Time (min) & 1.2 & 1.0 & 0.9 & 0.75 & 0.72 & 0.6 \\
\hline $\begin{array}{c}\text { SWIP (Lead Time / Takt } \\
\text { Time ) }\end{array}$ & 21.3 & 25.5 & 28.3 & 34.0 & 35.4 & 42.5 \\
\hline $\begin{array}{c}\text { How many chairs are } \\
\text { needed? (SWIP: Cycle } \\
\text { Time of Waiting (15min) } \\
\text { / Takt Time). }\end{array}$ & 12.5 & 15.0 & 16.7 & 20.0 & 20.8 & 25.0 \\
\hline
\end{tabular}

Abstract 24 Figure 2 Due to space constraints in post-vaccination waiting room to accommodate social distancing, calculations were needed to understand how many chairs would be needed with different volume/flow through the clinic (and ultimately was the rate limiting step in the total capacity of the clinic)

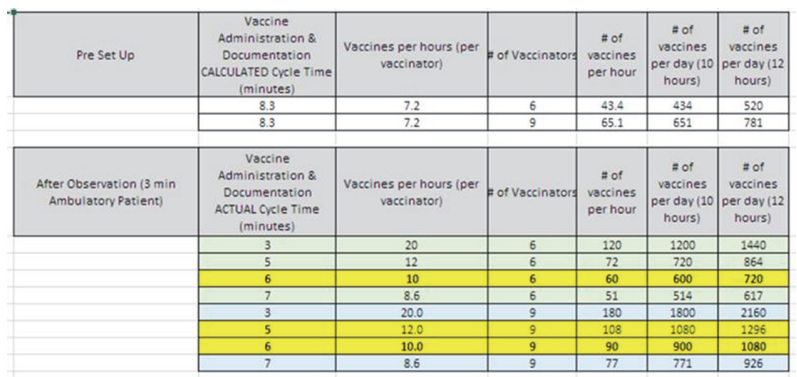

Abstract 24 Figure 3 Understanding cycle times and staffing needs at different rates of vaccinations per hour. Weighted average cycle times were used to accommodate differing complexity of patients (based on demographics of currently eligible populations). Based on the efficiency of cycle times achieved, different numbers of vaccinators and hours of operation would be needed to achieve volume goals

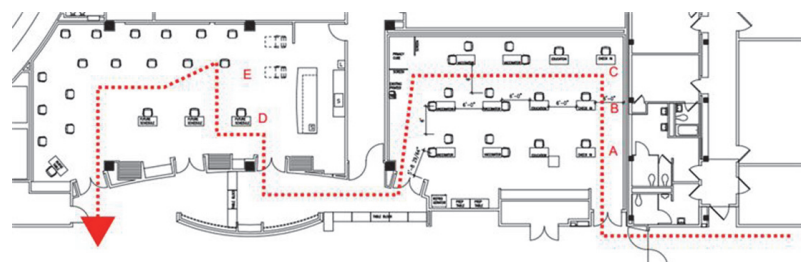

Abstract 24 Figure 4 Space planning: optimize flow, maximize capacity

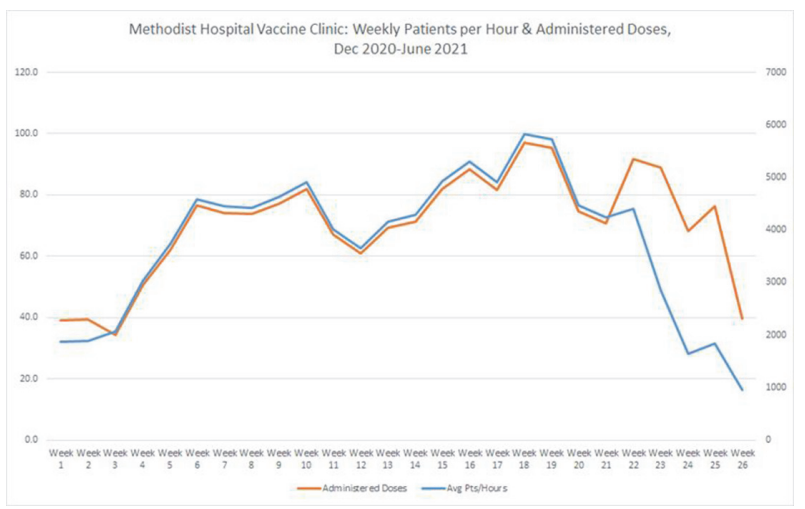

Abstract 24 Figure 5 Results, demonstrating rapid cycles of PDSA and frequent adjustments due to changing patient populations, vaccine supply, efficiencies achieved.

service in multiple languages, serving adult and pediatric populations, and ambulatory and non-ambulatory patients.

- We used predictive modeling to anticipate number of vials needed, standardized drawing process to maximize doses from each vial, and instituted a standby process to eliminate end-of-day waste.

Results In total, during a 6-month operation we administered nearly 100,000 doses.

Conclusions Our legacy as an organization with a mature culture of improvement contributed to trust of rapid cycles of PDSA, which allowed us to deliver on the Triple Aim:

- Reducing cost by using existing employees and volunteers

- Addressing health of the population by targeting multiple patient tiers to optimize supply

- Delivering experience from invite to injection with sensitivity to patient needs

\section{GETTING RID OF STUPID STUFF: ADDRESSING SYSTEM INEFFICIENCY THROUGH END USER FEEDBACK}

Jason Connelly, Lisa Gilley, Anna Sinclair. Novant Health

\subsection{6/bmjoq-2021-|HI.25}

Background Studies show that system inefficiency and processes that take away from the joy of medicine contribute to burnout. Using the Hawaii Pacific Health model, a program was established to allow for end user feedback concerning processes that contribute to workload.

Objectives Identify system processes and policies that increase work burden, produce redundancies, affect patient-safety, reduce satisfaction, or could be performed more efficiently by eliciting end user feedback. Provide evaluation of identified processes to then produce meaningful system change reducing workload where possible.

Methods Submissions were received by email or by entry using the Service Now platform from end users. Initial scope was for providers only with expansion to clinical administrators after 6 months. Requests were categorized by project team and variables assigned based on patient safety, provider burnout, similar prior requests, and whether the request could be completed quickly. Evaluation of each request was then directed by the project team with involvement from subject matter experts and other resources when necessary. Upon 
completion, project assessment remains necessary, resulted in education, removal, or change was completed by the project team. Evaluation of project was performed at 2 years post release.

Results During the initial 2-year project, 563 total requests were received with a closure rate of $97.9 \%$. Closed requests resulted in process change (25.9\%), removal $(0.6 \%)$, deemed necessary (40.7\%), education (32.78\%), and lack of response (2\%). Requests were categorized as relating to the EHR (70.9\%), pharmacy (2.3\%), documentation (4.9\%), compliance, $(1.8 \%)$, workflows $(6 \%)$, billing or coding $(2 \%)$, forms $(1.3 \%)$ and other (11.3\%).

Conclusions Programs to identify and evaluate processes that reduce the joy of healthcare can result in significant opportunities for change $(58 \%$ of requests in this project) via process change or elimination and education of team members on existing processes that are efficient.

\section{MEASURING PROFESSIONAL INTERPRETER USE AT THE IWK HEALTH CENTRE: A QUALITY IMPROVEMENT PROJECT}

Yasmeen Mansoor, Jeannette Comeau, Tania Wong. Dalhousie University and IWK Health Centre

\subsection{6/bmjoq-2021-IHI.26}

Background Patients with limited English proficiency (LEP) are subject to poorer health outcomes and improving interpreter access is a modifiable factor to improve health inequities faced by LEP patients.

Objectives To assess current interpreter provision for pediatric inpatients and their families at the IWK Health Centre by measuring the:

- proportion of non-English speaking patients identified at admission who received in-person interpretation during their hospital stay 